\title{
Disruption of mediator complex subunit 19 (Med19) inhibits cell growth and migration in tongue cancer
}

\author{
Li-Jun Zhu ${ }^{1,2}$, Wang-Xiang Yan ${ }^{3}$, Zhong-Wei Chen ${ }^{2}$, Yu Chen ${ }^{3}$, Dan Chen ${ }^{3}$, Tong-Han Zhang ${ }^{1}$ and Gui-Qing Liao ${ }^{1 *}$
}

\begin{abstract}
Background: Mediator complex subunit 19 (Med19) is a critical subunit of the mediator complex that forms a bridge between the transcription factors and RNA polymerase II. Although it has been reported that Med19 plays an important role in stabilizing the whole mediator complex, its biological importance in tongue cancer cell proliferation and migration has not been addressed.
\end{abstract}

Methods: By using MTT, BrdU incorporation, colony formation, flow cytometric, tumorigenesis and transwell assays, We tested the Med19 role on tongue cancer cell growth and migration.

Results: We demonstrated that lentivirus-mediated Med19 knockdown could arrest tongue cancer cells at G1 phase, inhibit tongue cancer cell proliferation and migration in vitro. The tumorigenicity of Med19 short hairpin RNA (shRNA)-expressing lentivirus infected tongue cancer cells were decreased after inoculating into nude mice.

Conclusions: These results indicate that Med19 plays an important role in tongue cancer proliferation and migration, and suggest possible applications for tongue cancer therapy.

Keywords: Med19, Tongue cancer, Tumorigenicity, Proliferation, Migration

\section{Background}

Oral squamous cell carcinoma represents the sixth most common cancer around the world [1], and tongue carcinoma is one of the most common types of oral cancer [2]. Similar to other malignancies, the formation of tongue carcinoma is a complex process associated with accumulation of genetic and epigenetic changes that occur during the progression of the disease. Understanding the molecular mechanisms underlying tongue carcinoma progression may provide unique strategies for the development of molecular-targeted therapies for prevention and treatment of tongue carcinoma. The Mediator complex is a large multiprotein complex which is vital for transcriptional regulation and controls cell proliferation and differentiation [3]. It is expressed ubiquitously and conserved from yeast to mammals [4]. In yeast, Mediator is composed of 25 subunits, and divided

\footnotetext{
* Correspondence: guiqingliao@126.com

'Department of Oral and Maxillofacial Surgery, Guanghua College of Stomatology, Sun Yat-Sen University, 56 Lingyuanxi Road, Guangzhou 510055, China

Full list of author information is available at the end of the article
}

into three discrete domains: the head, middle, and tail modules [5-7]. Med19 (ROX3) [Ensembl: ENSG00 000156603.10] was first identified during a screen for mutants with increased expression of the hemeregulated $\mathrm{CYC7}$ gene and proposed to be an transcriptional regulator because of its nuclear localization [4]. The hypothesis of Med19 (ROX3) as a transcriptional regulator was further confirmed in a gene expression microarray study in Med19 deletion strain because a broad range of genes was found to be up- or downregulated after Med19 functional disruption. Furthermore, Med19 has been demonstrated to be a component of the Mediator complex [8] and is essential for mediator binding and its activation of RNA Pol II $[9,10]$. Structural analysis showed that Med19 is involved in head-module subunits in mammalian mediator complex and plays an important role in the whole mediator stabilization.

The potent function of Med19 as a transcription coactivator for regulating gene expression pattern suggests its role in the development of malignancies. 
Recently, Med19 was reported to promote the proliferation of bladder cancer, hepatocellular carcinomas, prostate cancer, gastric cancer and breast cancer cells [11-15]. However, the functional role of Med19 in tongue cancer cell growth and migration has not been reported.

In the present study, we constructed recombinant lentivirus delivering short hairpin RNA (shRNA) against Med19, which expresses GFP as a marker. The effect of Med19 silencing in tongue cancer cell proliferation, tumor formation and cell migration was investigated in vitro and in vivo. Our investigation may gain more insights into the progression of tongue cancer and provide a new target for gene therapy for this lethal disease.

\section{Methods \\ Cell culture}

Tongue cancer Tca8113 cells and HEK293T cells were purchased from ATCC and maintained in DMEM supplemented with $10 \% \mathrm{FBS}$, at $37^{\circ} \mathrm{C}, 5 \% \mathrm{CO}_{2}$ (Gibco, Carlsbad, CA, USA).

\section{Med19 siRNA infection}

Med19 siRNA (5'-AAGGTGAAGGAGAAGCTAAGT-3') or negative control siRNA (5'- TTCTCCGAACGTGT CACGT-3') were inserted into pGCSIL-GFP lentiviral vector, respectively. The siRNA plasmids were transfected together into HEK293T cells with lentiviral helper plasmids to generate the respective lentiviruses using Lipofectamine 2000 (Invitrogen, Grand Island, NY 14072, USA). Viral stocks were made and used to infect tongue cancer cells. Cells were collected for mRNA and protein levels detection after $72 \mathrm{~h}$ after infection.

\section{Reverse transcription polymerase chain reaction}

Total mRNA samples of tongue cancer cells were prepared with Trizol reagent (Invitrogen, Grand Island, NY 14072, USA) according to the manufacturer's instructions. Samples $(2.0 \mu \mathrm{g})$ were used as templates to perform the RTPCR assay using M-MLV-RTase (Promega, Madison, WI 53711, USA). The resulting cDNA was amplified by using the SYBR-Green Master PCR Mix (Applied Biosystem, Grand Island, NY 14072, USA) in triplicates. Real-time PCR was performed on the TP800 qPCR System (Takara, SW, Akron, $\mathrm{OH} 44314$, USA). Primers used for real-time PCR were as follows: Actin-forward, 5'-GGCGGCACC ACCATGTACCCT-3', Actin-reverse, 5' -AGGGGCCGGA CTCGTCATACT-3'; Med19-forward, 5'-GTAACTTCC TGCCTGACCTG-3', Med19-reverse, TGTGCTTGTGC TTATTCTTCTTC-3'.

\section{Western blot analysis}

Cells were lysed in $1 \times$ SDS lysis buffer $(1 \mathrm{M}$ Tris- $\mathrm{HCl} \mathrm{pH}$ 6.8, 2\% SDS, 20\% glycerol, $1 \mathrm{mM}$ aprotinin, $1 \mathrm{mM}$ PMSF and $10 \mu \mathrm{g} / \mathrm{mL}$ leupeptin). The protein samples were separated by electrophoresis in SDS-PAGE and then transferred to a polyvinylidene difluoride (PVDF) membrane. After blocking with Tris buffer saline (TBS) containing 5\% nonfat milk and $0.1 \%$ Tween 20 overnight, the membrane was subsequently incubated with primary antibodies at room temperature for $2 \mathrm{~h}$ or at $4^{\circ} \mathrm{C}$ overnight and with secondary antibody for another $2 \mathrm{~h}$, respectively. The membrane was then developed using the ECL+plus ${ }^{\text {tix }}$ Western blotting system (Amersham).

\section{Cell proliferation assay}

Tca8113 cells were infected with Med19 siRNA lentivirus or control lentivirus for 3 days. About 2,000 cells were seeded into each well in 96-well plates. An MTT cell proliferation assay was performed for 5 consecutive days and a BrdU incorporation assay was performed at $24 \mathrm{~h}$ and $48 \mathrm{~h}$. Results were expressed as the absorbance at $570 \mathrm{~nm}$ and $490 \mathrm{~nm}$, respectively.

\section{Colony formation assay}

Med19 siRNA lentivirus or mock control infected Tca8113 cells were collected 3 days after lentivirus infection. For the plate clone forming experiment, 500 cells were mixed in culture medium, and seeded in 6-well plates and each with three duplicate wells. Afterward, the cells were incubated at $37^{\circ} \mathrm{C}$ in air with $5 \% \mathrm{CO}_{2}$ and the media were renewed every 3 days. Two weeks later, the colonies were stained with Giemsa and the colony number was statistically analyzed.

\section{Cell cycle analysis}

Lentivirus infected tongue cancer cells were fixed with $70 \%$ pre-chilled ethanol at $4^{\circ} \mathrm{C}$ for $1 \mathrm{~h}$ after 3 days of lentivirus infection. The fixed cells were washed and stained with propidium iodide (PI) mixture containing $50 \mu \mathrm{g} / \mathrm{mL}$ PI and $100 \mu \mathrm{g} / \mathrm{mL}$ ribonuclease in PBS for $45 \mathrm{~min}$ at $37^{\circ} \mathrm{C}$. The cells were passed through a 300-mesh nylon net before the DNA content was determined by quantitative flow cytometry with standard optics of FACScan flow cytometer (Becton-Dickinson FACSCalibur, San Jose, California, USA). All the groups were performed in triplet and statistically analyzed.

\section{Transwell migration assay}

To explore the role of Med19 in tongue cancer cells, we performed a transwell assay in a 24-well culture plate using the Cell Invasion Assay Kit. (Invitrogen, Grand Island, NY 14072, USA) Briefly, $1 \times 10^{4}$ cells from different groups were seeded on a fibronectin-coated polycarbonate membrane insert ( $6.5 \mathrm{~mm}$ in diameter with $8.0 \mu \mathrm{m}$ pores) in a transwell apparatus (Costar, Cambridge, MA, USA) and 
cultured in DMEM. FBS was added to the lower chamber. After incubation for $12 \mathrm{~h}$ at $37^{\circ} \mathrm{C}$ in a $\mathrm{CO}_{2}$ incubator, the cells on the top surface of the insert were removed by wiping with a cotton swab. Cells that migrated to the bottom surface of the insert were fixed with methanol, stained with crystal violet, and scored visually in 5 random fields using a light microscope.

\section{Xenograft model of tumor growth in vivo}

The study protocol was approved by the institutional ethics board of the Guanghua College of Stomatology, Sun Yat-Sen University. All efforts were made to minimize mice suffering. BALB/c nude mice (male, 4-6 weeks old, $20 \pm 2 \mathrm{~g}$ ) were purchased from Shanghai SLRC Experimental Animal Center. (Shanghai, P.R. China) Cells were resuspended at $2 \times 10^{7}$ cells $/ \mathrm{mL}$ and an aliquot of $0.25 \mathrm{~mL}$ cell suspension was injected subcutaneously into athymic nude mice. Tumor volumes were determined by external measurements and calculated according to the equation, $V=\left(L \times W^{2}\right) \times 0.52(\mathrm{~V}=$ volume, $\mathrm{L}=$ length and $\mathrm{W}=$ width) [16]. Mice were sacrificed after 22 days and tumor weights were measured.

\section{Statistical analysis}

All experiments were performed three times in triplicates. The data were analyzed with one-way ANOVA. Differences were considered statistically significant at $P$ value $<0.05$.

\section{Results \\ Transduction of Med19 shRNA into human tongue cancer cells}

Recombined lentivirus expressing Med19 shRNA (siMed19) or negative control shRNA (si-Negative) was transduced into human tongue cancer cells at the multiplicity of infection (MOI) of 100, and more than $90 \%$ of infected cells expressed GFP as determined with fluorescence microscopy 3 days post-transduction (Figure 1a). To determine the effect of RNAi on the expression of Med19 in tongue cancer cells, the mRNA and protein levels of Med19 were analyzed after 3 days of lentivirus infection, respectively. The cells transduced with Med19 shRNA showed lower expression of Med19 mRNA and protein than those with negative control shRNA or the blank control cells (Figure 1b, c).

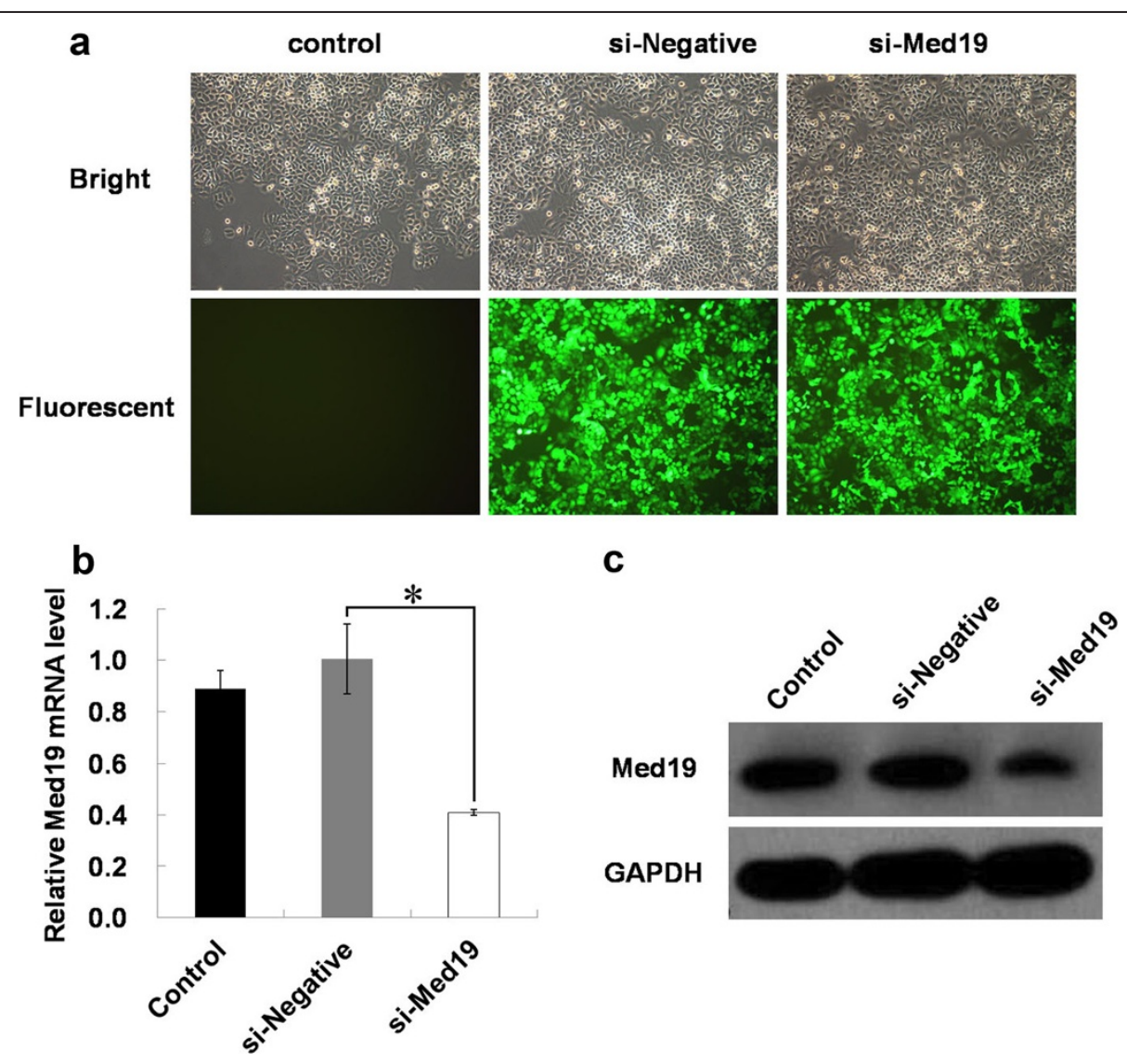

Figure 1 Med19 shRNA leads to down-regulation of Med19 in tongue cancer cells. (a) Blank Control (Control), nonsense siRNA (si-negtive) and Med19 shRNA (si-Med19) were transduced into tongue cancer cells. (b) The mRNA level of Med19 was downregulated by Med19 shRNA in tongue cancer cells. (c) The protein level of Med19 was downregulated by Med19 shRNA in tongue cancer cells. 


\section{Proliferation of tongue cancer cells is inhibited by Med19 shRNA}

We then detected whether Med19 shRNA affected the proliferation ability of tongue cancer cells by MTT cell proliferation assay and BrdU incorporation assay. As shown in Figure 2a, the growth pattern changed visibly from the second day to the fifth day in tongue cancer cells. Cells treated with Med19 shRNA-expressing lentivirus showed lower growth rate compared with the negative control shRNA or the blank control. To test whether silencing of Med19 could impede cell cycle progression, the incorporation rate of a proliferative marker, BrdU, in scrambled or Med19 siRNA-transduced cells was measured. Consistently, the BrdU assay also showed the growth of cells was inhibited $48 \mathrm{~h}$ after the cells were treated with Med19 shRNA-expressing lentivirus compared with negative control shRNA or blank controls (Figure 2b). These results revealed that knockdown of Med19 by shRNA in tongue cancer cells did have an inhibitory effect on cell growth and proliferation.

\section{The colony formation ability of tongue cancer cells is inhibited by Med19 shRNA}

We then further analyzed the proliferation potency of tongue cancer cells treatment with Med19 shRNA by cell colony formation assay. The colony number of Med19 shRNA treated cells decreased from $166.0 \pm 7.8$ colonies/ well in the control group to $124.0 \pm 4.4$ colonies/well in the Med19 shRNA lentivirus infected group in tongue cancer cells (Figure 2c, d).

\section{The cell cycle of tongue cancer cells is inhibited by Med19 shRNA}

We then performed flow cytometry assay to detect weather Med19 shRNA affected tongue cancer cell cycle by FACScalibur flow cytometer $72 \mathrm{~h}$ after transduction. The results showed that the proportion of tongue cancer cells in G1 phase increased significantly with the descrease of cells in the G2/M phase (Figure 2e, f). Our results thus suggested that Med19 shRNA inhibited tongue cancer cells proliferation through modulating cell cycle progression.

\section{The migration of tongue cancer cells is inhibited by Med19 shRNA}

To test whether tongue cancer cell migration was affected by Med19 shRNA, a transwell assay was performed. As showed in Figure 3a, when treated with Med19 shRNA, tongue cancer cell migration was inhibited. The migration rate was $31 \%$, whereas in the Med19-knockdown group, it was only $25 \%$ (Figure $3 \mathrm{~b}, P<0.05$ ). In this way, we could conclude that knockdown of Med19 could inhibit cell migration of tongue cancer cells. Med19 possibly plays a critical role in the migration of tongue cancer.
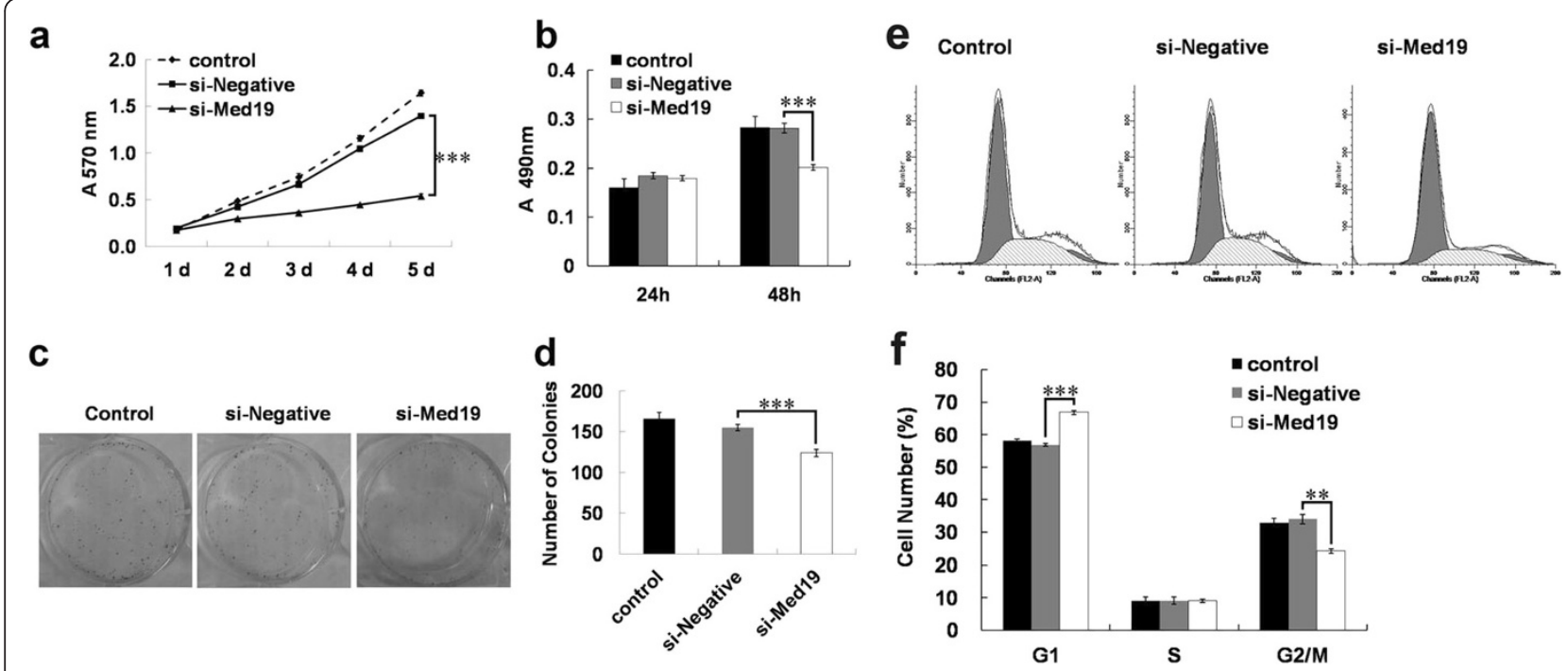

Figure 2 The proliferation of tongue cancer cells is inhibited upon Med19 shRNA treatment. (a) Growth curve by MTT assay. Cells were treated with negative control shRNA or Med19 shRNA and cultured for one to five days. (b) BrdU incorporation assay was performed on cells treated with Med19 shRNA or negative control shRNA. (c) Representative colony of tongue cancer cells treated with control shRNA or Med19 shRNA. (d) Cells treated with Med19 shRNA made less clone number compared with control shRNA or blank control. (e) Fluorescence-activated cell sorting (FACS) analysis of cells phase of tongue cancer cells treated with negative control or Med19 shRNA. (f) Cells treated with Med19 shRNA showed higher cell numbers at the G1 phase. Mean values are derived from three independent experiments. Errors indicate standard deviation. 


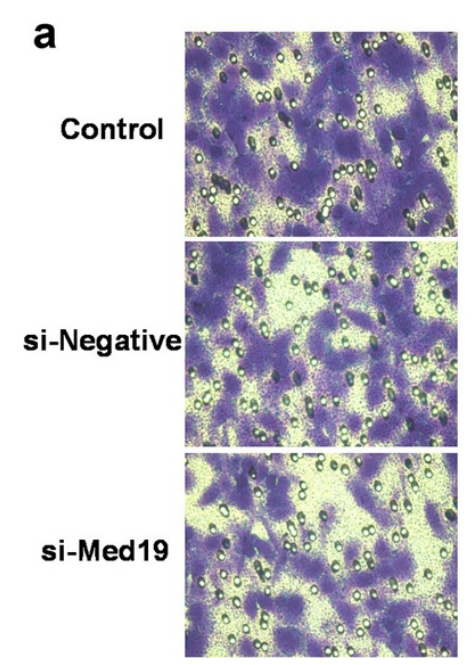

b

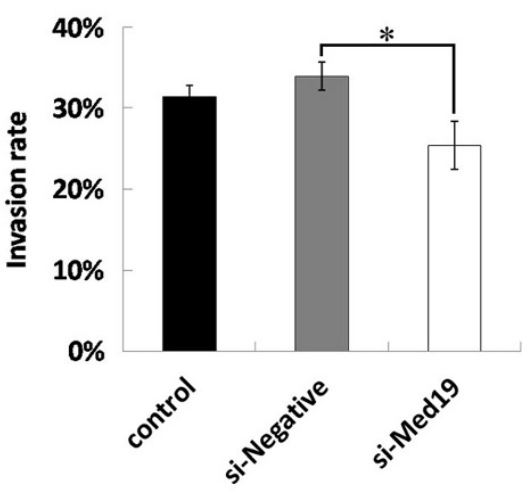

Figure 3 Med19 shRNA inhibit tongue cancer cell migration. (a) Pictures of crystal violet staining cells. (b) Med19 shRNA treated cells show a decrease in the number of migrated cells. Mean values are derived from three independent experiments. Errors indicate standard deviation.

The in vivo tumor growth of tongue cancer cells is inhibited by Med19 shRNA

To further explore the tumor inhibited ability of Med19 shRNA, we used a xenograft model to examine whether Med19 shRNA inhibited tumor growth in vivo (Figure 4a). When inoculated subcutaneously into athymus nude mice, tongue cancer cells treated with Med19 shRNA had dramatically reduced tumor volumes (Figure $4 \mathrm{~b}$ and $4 \mathrm{c}$ ) and tumor weights (Figure 4d) compared with blank control cells (Control) and control negative shRNA treated with cells, indicating that Med19 promotes the tumorigenesis of tongue cancer cells.

\section{Discussion}

Tongue carcinoma is one of the most common types of oral cancer [2], which represents the sixth most common cancer around the world [1]. Despite the improvements in treatment for tough cancer, the etiology of this disease is still unknown. With the help of lentivirusmediated gene silencing technique, more and more genes
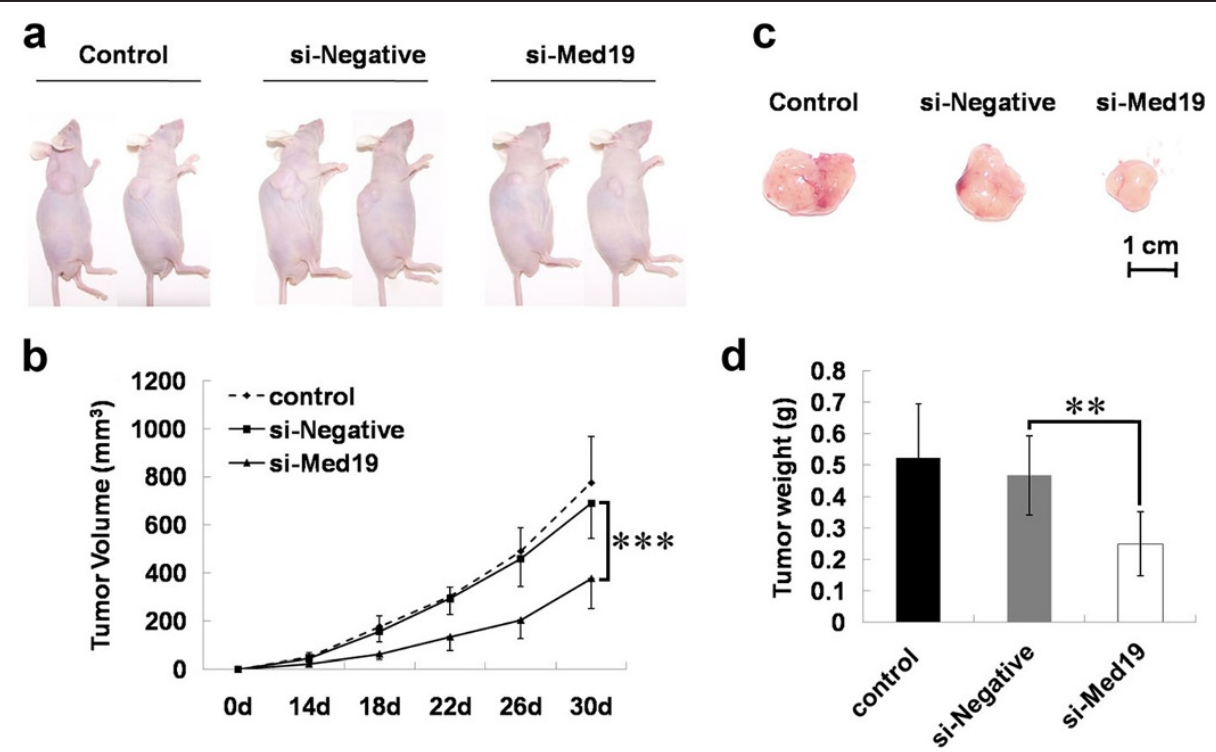

Figure 4 Med19 shRNA inhibits tongue cancer cell tumor growth in vivo. Representative images of tongue tumor-bearing mice (a) and tumors from mice $(\mathbf{c})$. (b) Med19 shRNA treated tongue cancer cells show smaller tumor volume. (d) Med19 shRNA treated tongue cancer cells show lower tumor weight. 
have been implicated to be associated with carcinogenesis in recent years. Among these cancer-related genes, many gene expression patterns have been demonstrated to be altered partially due to transcriptional regulation. Thus, elucidation of critical roles of transcriptional regulators will help us to understand the progression of tongue carcinogenesis.

Med19 is one of the components of the Mediator complex, which acts as a molecular bridge between transcriptional activator and RNA polymerase II [17]. Although Med19 has been suggested to play an important role in many cancer types [11-15], the pathologic importance of this molecule in tongue cancer is yet unknown. In this study, we investigated the antitumorigenic effects of lentivirus-mediated transduction of Med19 shRNA into tongue cancer cells by quantifying cellular proliferation, tumor growth, cell cycle and migration ability. Our results suggest that downregulation of Med19 by shRNA resulted in inhibition of cell proliferation, colony formation and migration ability, and induction of G0/G1 phase cell cycle arrest. Furthermore, the antitumor effects of Med19 were elucidated by in vivo tumorigenicity experiments. Our research was the first report in which it has been demonstrated that Med19 modulates tongue cancer cell proliferation and migration and disruption of Med19 has antitumorigenic effects.

The mechanism through which Med19 induced tongue carcinogenesis is still unknown. It has been reported that many components of the Mediator complex can directly bind a variety of regulatory transcription factors that are indispensible for cell growth and differentiation. For example, MED1/TRAP220 and MED17 can bind to GATA family membranes [18], BRCA-1 [19] and P53 [20]. Thus, it is possible that there may be interactions between Med19 and these cell growth transcription factors via direct or indirect binding. Udayakumar and his colleagues have showed that the Mediator complex regulates gene expression of Aurora-A, a centrosome kinase critical for cell cycle progression [21]. In prostate cancer, it has been demonstrated that the expression of CDK4 was decreased after Med19 disruption [15]. Overall, although it is possible that Med19 like other components of the Mediator complex, could directly or indirectly bind with transcription factors, which are necessary for cell growth and cell cycle progression, it is still worth working to demonstrate the exact signaling pathway involved in the regulation of progression in tongue cancer by Med19.

RNA interference (RNAi) is sequence-specific posttranscriptional gene silencing by short small interfering RNA (siRNA), which is thought to be a powerful approach for studying gene function and gene therapy [22]. Due to the ease of delivery, adenovirus, retrovirus, and lentivirus are commonly used for shRNA transduction [23-25]. Lentiviruses are considered to be more suitable for gene therapy for their safety and life-long expression of shRNA $[26,27]$.

\section{Conclusions}

Our results provide the evidence that lentivirusmediated Med19 downregulation inhibits tongue cancer cell proliferation and tumorigenesis both in vitro and in vivo, suggesting that disruption of Med19 by lentivirus transduction may be a promising approach for tongue cancer therapy.

\section{Abbreviations}

Med19: Mediator complex subunit 19; FACS: Fluorescence-activated cell sorting; MOI: Multiplicity of infection; PVDF: Polyvinylidene difluoride; RT-PCR: Reverse transcription polymerase chain reaction; shRNA: Short hairpin RNA.

\section{Competing interests}

The authors declare that they have no competing interests.

\section{Authors' contributions}

All authors read and approved the final manuscript.

\section{Author details}

'Department of Oral and Maxillofacial Surgery, Guanghua College of Stomatology, Sun Yat-Sen University, 56 Lingyuanxi Road, Guangzhou 510055, China. ${ }^{2}$ Department of Oral and Maxillofacial Surgery, Guangdong Academy of Medical Sciences/Guangdong General Hospital, 106 Zhongshan 2nd Rd, Guangzhou 510080, China. ${ }^{3}$ Department of Oral and Maxillofacial Surgery, First Affiliated Hospital, Sun Yat-Sen University, 58 Zhongshan 2nd Rd, Guangzhou 510055, China.

Received: 26 October 2012 Accepted: 9 May 2013

Published: 27 May 2013

\section{References}

1. Jemal A, Siegel $R, X u J$, Ward E: Cancer statistics, 2010. CA Cancer J Clin 2010, 60:277-300.

2. Jemal A, Siegel R, Ward E, Hao Y, Xu J, Murray T, Thun MJ: Cancer statistics, 2008. CA Cancer J Clin 2008, 58:71-96.

3. Myers LC, Kornberg RD: Mediator of transcriptional regulation. Annu Rev Biochem 2000, 69:729-749.

4. Rosenblum-Vos LS, Rhodes L, Evangelista CC Jr, Boayke KA, Zitomer RS: The ROX3 gene encodes an essential nuclear protein involved in CYC7 gene expression in Saccharomyces cerevisiae. Mol Cell Biol 1991, 11:5639-5647.

5. Boube M, Joulia L, Cribbs DL, Bourbon HM: Evidence for a mediator of RNA polymerase II transcriptional regulation conserved from yeast to man. Cell 2002, 110:143-151.

6. Beve J, Hu GZ, Myers LC, Balciunas D, Werngren O, Hultenby K, Wibom R, Ronne $\mathrm{H}$, Gustafsson CM: The structural and functional role of Med5 in the yeast Mediator tail module. J Biol Chem 2005, 280:41366-41372.

7. Singh H, Erkine AM, Kremer SB, Duttweiler HM, Davis DA, lqbal J, Gross RR, Gross DS: A functional module of yeast mediator that governs the dynamic range of heat-shock gene expression. Genetics 2006, 172:2169-2184

8. Sato S, Tomomori-Sato C, Banks CA, Parmely TJ, Sorokina I, Brower CS, Conaway RC, Conaway JW: A mammalian homolog of Drosophila melanogaster transcriptional coactivator intersex is a subunit of the mammalian Mediator complex. J Biol Chem 2003, 278:49671-49674.

9. Baidoobonso SM, Guidi BW, Myers LC: Med19 (Rox3) regulates intermodule interactions in the Saccharomyces cerevisiae mediator complex. J Biol Chem 2007, 282:5551-5559.

10. Ding N, Tomomori-Sato C, Sato S, Conaway RC, Conaway JW, Boyer TG: MED19 and MED26 are synergistic functional targets of the RE1 silencing transcription factor in epigenetic silencing of neuronal gene expression. J Biol Chem 2009, 284:2648-2656. 
11. Li LH, He J, Hua D, Guo ZJ, Gao Q: Lentivirus-mediated inhibition of Med19 suppresses growth of breast cancer cells in vitro. Cancer Chemother Pharmacol 2011, 68:207-215.

12. Zhang $H$, Jiang $H$, Wang W, Gong J, Zhang L, Chen Z, Ding Q: Expression of Med19 in bladder cancer tissues and its role on bladder cancer cell growth. Urol Oncol 2011, 30:920-927.

13. Zou SW, Ai KX, Wang ZG, Yuan Z, Yan J, Zheng Q: The role of Med19 in the proliferation and tumorigenesis of human hepatocellular carcinoma cells. Acta Pharmacol Sin 2011, 32:354-360.

14. Ding XF, Huang GM, Shi Y, Li JA, Fang XD: Med19 promotes gastric cancer progression and cellular growth. Gene 2012, 504:262-267.

15. Cui X, Xu D, Lv C, Qu F, He J, Chen M, Liu Y, Gao Y, Che J, Yao Y, Yu H: Suppression of MED19 expression by shRNA induces inhibition of cell proliferation and tumorigenesis in human prostate cancer cells. BMB Rep 2011, 44:547-552.

16. Khor TO, Keum YS, Lin W, Kim JH, Hu R, Shen G, Xu C, Gopalakrishnan A, Reddy $\mathrm{B}$, Zheng $\mathrm{X}$, Conney AH, Kong AN: Combined inhibitory effects of curcumin and phenethyl isothiocyanate on the growth of human PC-3 prostate xenografts in immunodeficient mice. Cancer Res 2006, 66:613-621.

17. Lewis BA, Reinberg D: The mediator coactivator complex: functional and physical roles in transcriptional regulation. J Cell Sci 2003, 116:3667-3675.

18. Crawford SE, Qi C, Misra P, Stellmach V, Rao MS, Engel JD, Zhu Y, Reddy JK: Defects of the heart, eye, and megakaryocytes in peroxisome proliferator activator receptor-binding protein (PBP) null embryos implicate GATA family of transcription factors. J Biol Chem 2002, 277:3585-3592.

19. Wada O, Oishi H, Takada I, Yanagisawa J, Yano T, Kato S: BRCA1 function mediates a TRAP/DRIP complex through direct interaction with TRAP220. Oncogene 2004, 23:6000-6005.

20. Frade $R$, Balbo $M$, Barel M: RB18A, whose gene is localized on chromosome 17q12-q21.1, regulates in vivo p53 transactivating activity Cancer Res 2000, 60:6585-6589.

21. Udayakumar TS, Belakavadi M, Choi KH, Pandey PK, Fondell JD: Regulation of Aurora-A kinase gene expression via GABP recruitment of TRAP220/ MED1. J Biol Chem 2006, 281:14691-14699.

22. Castanotto D, Rossi JJ: The promises and pitfalls of RNA-interference -based therapeutics. Nature 2009, 457:426-433.

23. Xia $\mathrm{H}, \mathrm{Mao} \mathrm{Q}$, Paulson $\mathrm{HL}$, Davidson BL: siRNA-mediated gene silencing in vitro and in vivo. Nat Biotechnol 2002, 20:1006-1010.

24. Brummelkamp TR, Bernards R, Agami R: Stable suppression of tumorigenicity by virus-mediated RNA interference. Cancer Cell 2002, 2:243-247.

25. Qin XF, An DS, Chen IS, Baltimore D: Inhibiting HIV-1 infection in human T cells by lentiviral-mediated delivery of small interfering RNA against CCR5. Proc Natl Acad Sci USA 2003, 100:183-188.

26. ter Brake O, Konstantinova P, Ceylan M, Berkhout B: Silencing of HIV-1 with RNA interference: a multiple shRNA approach. Mol Ther 2006, 14:883-892.

27. Manjunath N, Wu H, Subramanya S, Shankar P: Lentiviral delivery of short hairpin RNAs. Adv Drug Deliv Rev 2009, 61:732-745.

doi:10.1186/1477-7819-11-116

Cite this article as: Zhu et al:: Disruption of mediator complex subunit 19 (Med19) inhibits cell growth and migration in tongue cancer. World Journal of Surgical Oncology 2013 11:116.

\section{Submit your next manuscript to BioMed Central and take full advantage of:}

- Convenient online submission

- Thorough peer review

- No space constraints or color figure charges

- Immediate publication on acceptance

- Inclusion in PubMed, CAS, Scopus and Google Scholar

- Research which is freely available for redistribution

Submit your manuscript at www.biomedcentral.com/submit
C Biomed Central 